\title{
Misconceptions About the Evolution of Complexity
}

\author{
Andrew J. Petto • Louise S. Mead
}

Published online: 27 September 2008

(C) Springer Science + Business Media, LLC 2008

\begin{abstract}
Despite data and theory from comparative anatomy, embryology, molecular biology, genomics, and evolutionary developmental biology, antievolutionists continue to present the eye as an example of a structure too complex to have evolved. They stress what we have yet to explain about the development and evolution of eyes and present incomplete information as evidence that evolution is a "theory in crisis." An examination of the evidence, however, particularly evidence that has accumulated in the twentieth and twenty-first centuries, refutes antievolutionists' claims. The distribution of eyes in extant organisms, combined with what we now know about the control of eye development across diverse groups of organisms, provides significant evidence for the evolution of all major components of the eye, from molecular to morphological, and provides an excellent test of predictions based on common ancestry.
\end{abstract}

Keywords Evolution of complexity · Vertebrate eye ·

Misconceptions · Intelligent design

Creationists frequently quote Darwin on the improbability that natural selection could produce the vertebrate eye:

To suppose that the eye, with all its inimitable contrivances for adjusting the focus to different distances, for admitting different amounts of light, and for the

\footnotetext{
A. J. Petto

Department of Biological Sciences, University of Wisconsin, PO Box 413, Milwaukee, WI 53201-0413, USA

L. S. Mead $(\bowtie)$

National Center for Science Education,

PO Box 9477, Berkeley, CA 94709-0477, USA

e-mail: mead@ncseweb.org
}

correction of spherical and chromatic aberration, could have been formed by natural selection, seems, I freely confess, absurd in the highest possible degree (Darwin 1859, p. 186).

Yet they tend to overlook the rest of the paragraph, in which Darwin reassures the reader that natural selection is quite up to the task:

Yet reason tells me, that if numerous gradations from a perfect and complex eye to one very imperfect and simple, each grade being useful to its possessor, can be shown to exist; if further, the eye does vary ever so slightly, and the variations be inherited, which is certainly the case; and if any variation or modification in the organ be ever useful to an animal under changing conditions of life, the difficulty of believing that a perfect and complex eye could be formed by natural selection, though insuperable by our imagination, can hardly be considered real. (Darwin 1859, p. 186)

Darwin openly confronted the main doubt about biological evolution troubling the general public both in his day and ours: the appearance of design in complex biological organisms. Because Darwin's contemporaries were familiar with William Paley's proposal that the extraordinary complexity of vertebrate eyes could only be explained by divine design, Darwin chose the eye as an example of biological complexity that could in fact be explained through natural processes (Gray 1876). He suggested an examination of the structure and function of light-sensing organs in nonvertebrate lineages, noting that invertebrates show great variation in the form and function of their eyes and "numerous gradations of structure" (Darwin 1859, p. 187). Indeed, an examination of eyes in various mollusks reveals each of the steps hypothesized in the evolution of 
complex eyes can be found fully functioning in extant species, following Darwin's prediction. [Complexity Evolves http://www.expelledexposed.com/videos.php]

To anyone open to Darwin's evidence, based on data from comparative anatomy (within and among taxa), comparative ecology (adaptation of related organisms to local environments), and the fossil record, augmented by twentieth and twenty-first century discoveries in comparative embryology, molecular biology, and developmental studies, it is clear that natural selection is a powerful process capable of producing highly complex biological structures. Yet, antievolutionists continue to insist that eyes could not have evolved in a stepwise fashion and therefore must have been purposely designed. This perspective is especially evident in intelligent design instructional materials (e.g., Meyer et al. 2007).

Since the complexity of vertebrate eyes is a common antievolution argument, let us look at what Darwin called an "organ of extreme perfection" and see how evolution accounts for it. Readers interested in a detailed discussion of the evolution of complex features should consult Gregory (2008), and those seeking a comprehensive overview of the state of our current knowledge of eye evolution should consult Lamb et al. (2007, 2008), Buschbeck and Friedrich (2008), Cronin and Porter (2008), Oakley and Pankey (2008), Piatigorsky (2008), and Serb and Eernisse (2008).

\section{What is "the Eye"?}

Like most organs, vertebrate eyes consist of several different tissues that contribute to form and function. Although these are integrated into a single functional complex that we call "the eye," it is clear from developmental and anatomical studies that eyes consist of modular components: eyes resemble a prefabricated building - with many elements built independently and then assembled into a whole. One important feature of modularity for eye evolution is that the genes, biochemical pathways, and even the final assembly of components can be modified by natural selection individually or collectively (Franz-Odendaal and Hall 2006; Oakley 2003). The result is a wealth of diversity of eye types resulting from the great number of possibilities for combining several simpler components in a variety of ways.

\section{Evolution and Eye Variation}

Darwin noted that there was considerable variation among taxa in the form of eyes, and modern researchers agree: over $96 \%$ of animal species have eyes capable of generating signals good enough to form an image, though the forms that eyes take vary greatly (Fernald 2006). What can variation in the form of eyes tell us about their evolution? For one thing, all vertebrates appear to have the same photosensitive molecules in the retina (Fernald 2006; Santillo et al. 2006), and the retina and lens appear to form in the same ways from the same embryonic tissues (Lamb et al. 2007; Shimeld et al. 2005). Shimeld et al. (2005) report that the form of crystallin in the vertebrate lens is distinct from crystallin in urochordates but that the gene encoding this protein is only slightly modified from an ancestral gene in the early chordates. They also report that the gene functions the same way in both branches of the chordates, where it acts to integrate the lenticular and retinal components of eyes.

These studies tell us that vertebrate eyes emerged early in the history of our lineage, that their various components are modified from preexisting structures, and that many developmental, structural, and functional aspects of vertebrate eyes are limited to this evolutionary lineage. Indeed, Shimeld et al. (2005) suggest that the finer discrimination of visual stimuli made possible by vertebrate eyes was a key variable establishing vertebrates as a new evolutionary lineage.

\section{Evolution and Eye Function}

The scientific analysis of genetic developmental pathways and of the biochemical constitution of the visual apparatus helps answer Darwin's basic questions about how these biological entities vary across taxa and tells us much about the evolution of eyes. One key variable in the development of vertebrate eyes is the emergence of a light-sensitive molecule that can cause a cell to generate a signal. It turns out that the photosensitive compounds found in animal retinas - collectively known as opsins - are widespread among living organisms. Two primary groups of vertebrate opsins are rhodopsins, found in the rod photoreceptor cells, and photopsins (or cone opsins), found in the cones (Gehring and Ikeo 1999; Béjà et al. 2001). Rhodopsins and cone opsins (photopsin I, photopsin II, etc.) are composed of a protein moiety opsin and the cofactor retinal which is composed of a photoreactive chromophore and is the light-responsive part of the opsin complex (Fernald et al. 2006; Santillo et al. 2006). Response to various wavelengths of light is achieved by attaching retinal to the different opsin molecules. This variation results in an assortment of receptors that support adaptation to different environments: for example, tricolor vision in humans and other anthropoid primates (Lamb et al. 2007). Opsins also occur in organisms without eyes, allowing these organisms to sense and respond to light, even when there are no image-forming organs (Santillo et al. 2006). 


\section{Evolution and Eye Development}

Developmental biology helps us to understand the evolution of eyes in two main ways: (1) it shows us how the basic components are constructed and then assembled; and (2) it shows us how different configurations of these components can provide useful sensory information about the environment and thus can be subject to natural selection. What these studies also tell us is that the "directions" that genes provide to cells are modified by the "directions" that other genes generate.

Both vertebrates and invertebrates use the developmental gene Pax6 to create eyes (Gehring and Ikeo 1999). What makes vertebrate and invertebrate eyes different is the context in which the gene operates. Studies of the Pax6 gene make it clear that the output of this gene is modified in different lineages by the presence of other genes and developmental pathways. Gehring and Ikeo (1999) found that the Pax6 gene assembles the basic photosensitive "cell assembly" which is then incorporated into different arrangements such as the simple, single-chambered eyes found in vertebrates or the compound eyes found in insects. The presence and role of Pax6 in structurally different types of eyes, from the compound eyes of a fruit fly to the single-lens camera eyes of a human, indicate genetic and developmental similarities best explained by common descent. What is more, Kozmik (2008) points out that there is a family of Pax genes in living organisms that have neither eyes nor a connection between photoreceptors and the nervous system, so the Pax6 gene itself has probably been modified from other genes associated with producing cells capable of responding to light.

Finally, developmental studies give us a more complete answer to the rhetorical question, "What good is half an eye?" This creationist argument assumes that only fully functional eyes would provide a selective advantage to an organism. Yet, any ability an organism had to gather visual information from the environment would confer a selective advantage over those organisms that lack such information. Just being able to tell light from dark is an advantage in many settings, and the ability to detect the direction of the light source is even more useful. There are close relatives of vertebrates that lack some of the components of "typical" vertebrate eyes, such as the hagfish (Lamb et al. 2007); while a hagfish cannot read or play video games, its "incomplete" eyes are still quite functional and provide a selective advantage in its environment, indicating that eyes can form even without selection for an image-forming organ. Moreover, developmental studies show that the assembly of the parts of vertebrate eyes that $d o$ exist in hagfishes are shared with other vertebrates, and eyes that emerged later in the evolutionary history of the vertebrates were built on this common foundation.

\section{Conclusions}

So, when we consider the claims that vertebrate eyes are a challenge to evolution, we see that modern evolutionary science refutes it. Contemporary research tells us:

1. That the step by step construction of vertebrate eyes is no mystery: eyes are complex structures resulting from the assembly of less complex modules;

2. That the basic chemistry (structure and function) of photosensitive molecules is the same whether the cells that contain these compounds are connected to the nervous system or not;

3. That the existing variations in the appearance of the component structures that support light detection show the ways in which intermediate features would be (and in fact are) useful to the organisms that possess them;

4. That questions about the plausibility of the evolution of complex structures such as eyes are due to a lack of data, not a failure of evolutionary theory, and that the basic roadmap that Darwin set out for resolving the difficulties has indeed led us to the solutions.

There is still much to learn about eye evolution. Lamb et al. (2007) lay out new hypotheses to be tested that will resolve some of the remaining questions about this fascinating subject but leave no doubt that complex structures such as the eyes could have arisen through natural processes by many small steps rather than requiring a single large step as design proponents hold. Darwin provided explicit predictions for the intermediate steps in the evolution of eyes, observable in extant organisms - and we now know much more about the genetics and physiology of these steps. In contrast to the claims of antievolutionists, what we know today about the phylogeny, comparative anatomy, development, genetics, and function of vertebrate (and other) eyes is a triumph, not a failure, of evolutionary science and of Darwin's original vision of descent with modification.

\section{References}

Béjà $\mathrm{O}$, Spudich EN, Spudich JL, Leclerc M, DeLong EF. Proteorhodopsin phototrophy in the ocean. Nature 2001;411:786-9. doi: $10.1038 / 35081051$.

Buschbeck E, Friedrich M. Evolution of insect eyes: tales of ancient heritage, deconstruction, reconstruction remodeling, and recycling. Evolution. Educ Outreach. 2008;1(4). doi:10.1007/s12052008-0086-z.

Cronin TW, Porter ML. The good, the bad, and the ugly: the evolution of crustacean compound eyes. Evolution. Educ Outreach. 2008; 1 (4). doi:10.1007/s12052-008-0085-0. 
Darwin CR. On the origin of species by means of natural selection, or the preservation of favoured races in the struggle for life. London: Murray; 1859.

Fernald RD. Casting a genetic light on the evolution of eyes. Science. 2006;313:1914-8. doi:10.1126/science.1127889.

Franz-Odendaal TA, Hall BK. Modularity and sense organs in the blind cavefish, Astyanax mexicanus. Evol Dev. 2006;8(1):94100. doi:10.1111/j.1525-142X.2006.05078.x.

Gehring WJ, Ikeo K. Pax6: mastering eye morphogenesis and eye evolution. Trends Genet. 1999;15(9):371-7. doi:10.1016/S01689525(99)01776-X.

Gray A. Darwiniana: essays and reviews pertaining to Darwinism. New York: D. Appleton; 1876.

Gregory TR. The evolution of complex organs. Evolution. Educ Outreach. 2008;1(4). doi:10.1007/s12052-008-0076-1.

Kozmik Z. The role of Pax genes in eye evolution. Brain Res Bull. 2008;75:335-9. doi:10.1016/j.brainresbull.2007.10. 046.

Lamb TD, Collin SP, Pugh EN Jr. Evolution of the vertebrate eye: opsins, photoreceptors, retina, and eye cup. Nat Rev Neurosci. 2007;8:960-75. doi:10.1038/nrn2283.

Lamb TD, et al. The origin of the vertebrate eye. Evolution. Educ Outreach. 2008;1(4). doi:10.1007/s12052-008-0091-2.
Meyer SC, Minnich S, Moneymaker J, Nelson PA, Seelke R. Explore evolution: the arguments for and against neo-Darwinism. Melbourne: Hill House; 2007.

Oakley TH. The eye as a replicating and diverging, modular developmental unit. Trends Ecol Evol. 2003;18(12):623-7. doi:10.1016/j.tree.2003.09.005.

Oakley TH, Pankey MS. Opening the "black box": the genetic and biochemical basis of eye evolution. Evolution. Educ Outreach. 2008;1(4). doi:10.1007/s12052-008-0090-3.

Piatigorsky J. A genetic perspective on eye evolution: gene sharing, convergence and parallelism. Evolution. Educ Outreach. 2008; 1(4). doi:10.1007/s12052-008-0077-0.

Santillo S, Orlando P, De Petrocelis L, Cristino L, Guglielmotti V, Musio C. Evolving visual pigments: hints from the opsin-based proteins in a phylogenetically old "eyeless" invertebrate. Biosystems. 2006;86:3-17. doi:10.1016/j.biosystems.2006.03.008.

Serb JM, Eernisse DJ. Charting evolution's trajectory: using molluscan eye diversity to understand parallel and convergent evolution. Evolution. Educ Outreach. 2008;1(4). doi:10.1007/s12052-008-0084-1.

Shimeld SM, Purkiss AG, Dirks RPH, Bateman OA, Slingsby C, Lubsen NH. Urochordate $\beta \gamma$-crystalline and the evolutionary origin of the vertebrate eye lens. Curr Biol. 2005;15:1684-9. doi:10.1016/j.cub.2005.08.046. 